\title{
Security scare puts Pluto launch at risk
}

\section{Geoff Brumfiel, Washington}

A NASA mission to Pluto could arrive five years late and cost tens of millions of dollars more because of a security clamp-down at Los Alamos National Laboratory in New Mexico.

The nuclear-powered New Horizons spacecraft, scheduled for launch in 2006, should be fuelled by plutonium from a reprocessing facility at Los Alamos. But work at the lab shut down on 16 July, pending a security review into the disappearance of two classified computer-storage devices (see Nature 430, 387; 2004). If the reprocessing facilities are not restarted soon, the mission could miss its launch window. "We're taking this very seriously," says Alan Stern, principal investigator of the project and director of the space-studies department at the Southwest Research Institute in Boulder, Colorado.

New Horizons is a $\$ 720$-million spacecraft designed to look at the Solar System's farthest planet and the Kuiper belt: a ring of icy objects beyond Neptune's orbit. Far from the Sun, the craft will depend on nuclear power to run its scientific instruments.

Under an agreement with NASA, Los Alamos is to produce 36 plutonium fuel elements. Together with 36 spare elements from

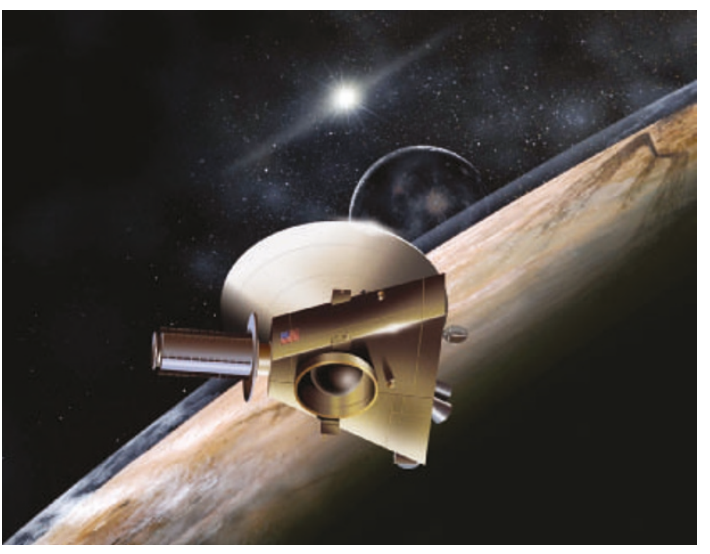

The New Horizons probe is rapidly running out of time.

the Cassini mission to Saturn, these will power the spacecraft on its nine-year journey to Pluto, says Orlando Figueroa, deputy associate administrator for programmes at NASA's Science Mission Directorate in Washington DC. To keep the satellite on schedule for the January 2006 launch date, the lab must deliver all the elements by the end of the year.

But on 4 August, Los Alamos director Peter Nanos said that it would take roughly two months for work at the lab to resume. Mission planners are racing to figure out how to work the delay into their schedule.
The two-week launch window in January 2006 is important because it would allow the craft to bounce off Jupiter's gravitational field on its way to Pluto. New Horizons could potentially launch in February 2007, but it would not be able to use Jupiter as a springboard and so would face an additional three to four years of travel time. Contingency plans drawn up by NASA put the extra costs, such as the need to run a mission control centre for longer than expected, at $\$ 67$ million.

In the coming months, mission planners will consider the implications of redesigning the mission so that it uses fewer fuel pellets. "People are looking at lots and lots of remedies," says Stern. Adding batteries that can be used to power up instruments when the craft arrives at Pluto is one possibility. Another is that some of the spacecraft's eight science instruments could be removed, says Figueroa.

The fuel shortage is the most pressing problem currently facing the mission, but it is by no means the only challenge. Figueroa says that the launch rocket still needs final approval. And because the mission uses nuclear power, the launch-approval process is more lengthy than normal. "This is a lesson in risk management," he says.

\section{Doped athletes flex muscles against drug company}

\section{Karoline Schwarzberg, Munich}

Supporters of athletes who received steroids under East Germany's state-controlled doping programme are turning up the heat on the pharmaceutical company alleged to have supplied the drugs.

Pressure on Jenapharm, based in Jena, Thuringen, has been mounting since July 2003, when a former employee alleged on television that the company supplied sports scientists in the German Democratic Republic (GDR) with substances used in the doping programme. Rainer Hartwich, who now works as a physician and gives medical advice to victims of doping, also admitted to having been involved in confidential clinical doping research.

Jenapharm's management disputes the allegations, saying the company produced the drugs for medical purposes and was not involved in doping studies - a claim that will now be examined by a prominent German molecular biologist.

The extent of doping is unknown, but thousands of athletes are thought to have been given muscle-boosting steroids during the 1970s and 1980s. Some, such as Olympic long-jumper and sprinter Heike Drechsler, say they were told these were vitamins. Steroids have many side effects, including an increased risk of cancer and infertility.

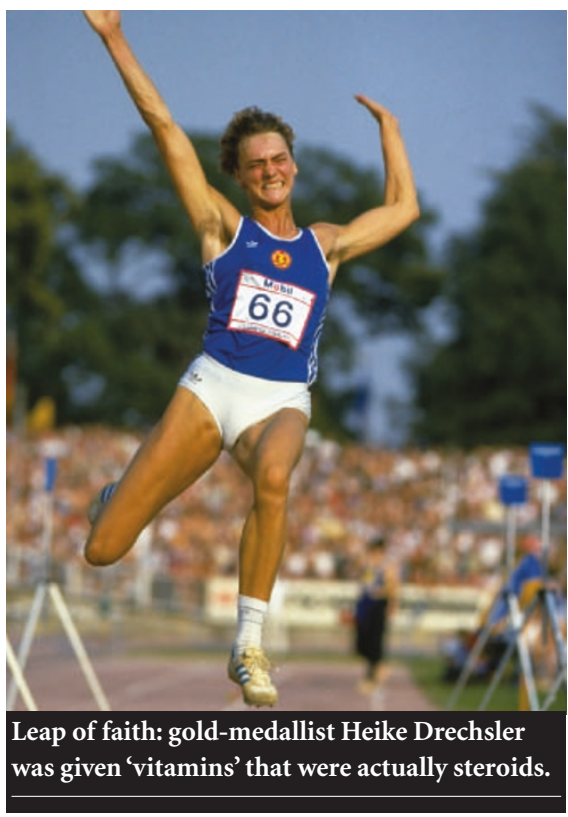

Help for Victims of Doping, a Weinheimbased organization that supports athletes from the former GDR, is urging Jenapharm to pay several million euros in compensation to the group of victims. It also accuses Jenapharm, which was taken over by Berlinbased drug company Schering in 2001, of refusing to open its archives so the extent of its involvement can be established. The firm did not respond to requests for an interview.

Last month, the society enlisted the help of Werner Franke, a prominent molecular biologist from the University of Heidelberg. In the early 1990s, Franke was on a commission of the Wissenschaftsrat, Germany's influential science council, that investigated people responsible for doping. He is married to Brigitte Berendonk, an athlete who left East Germany in 1958.

During previous investigations, Franke and Berendonk unearthed documents that he is using to compile a report on Jenapharm's involvement. The report, due at the end of August, could make uncomfortable reading for the company, as Franke claims he will be able to provide evidence of Jenapharm's links to the doping programme. 